\title{
3 Research Soure \\ Near Infra-Red Labelling and Tracking of Transplanted Corneal Endothelial Graft
}

\section{Maninder Bhogal}

Guy's \& St Thomas'

\section{Heng-Pei Ang}

Singapore Eye Research Institute

Shu-Jun Lin

Singapore Eye Research Institute

\section{Lwin Chan}

Singapore Eye Research Institute

\section{Khadijah Adnan}

Singapore Eye Research Institute

Gary Peh ( $\nabla$ gary.peh.s.I@seri.com.sg )

Singapore Eye Research Institute

Jodhbir Mehta

Singapore National Eye Center

\section{Research Article}

Keywords: corneal transplantation, in vivo imaging, pre-clinical animal model, 1,1'-dioctadecyl-3,3,3',3'tetramethylindotricarbocyanine iodide (DIR)

Posted Date: March 10th, 2021

DOl: https://doi.org/10.21203/rs.3.rs-244628/v1

License: (c) (1) This work is licensed under a Creative Commons Attribution 4.0 International License.

Read Full License 


\section{Abstract}

Following corneal transplantation, there is an initial decline in corneal endothelial cells (CECs) following graft preparation and surgery. Monitoring post-transplantation is only possible months after surgery by specular microscopy, which has a limited field of view. We have developed a labelling approach using 1,1'-dioctadecyl-3,3,3',3'-tetramethylindotricarbocyanine iodide (DIR) dye solution, that enabled tracking of labelled CECs in vivo for at least one month. Initial in vitro optimization of dye concentration, cellular toxicity and real-time cell migration was assessed using propagated primary CECs. Subsequent in vivo evaluation of cellular labelling was assessed within a rabbit wound healing model. Finally, real-time visualization of human cadaver donor tissue incubated in DIR transplanted into rabbits was achieved using the Heidelberg Spectralis. Results revealed detectable fluorescence increased with concentration to a plateau of $100 \mu \mathrm{g} / \mathrm{ml}$, with no toxicity of CECs at any concentration evaluated. DIR-labelled CECs were detectable in vivo upto 1 month, and transplanted labelled donor graft could be visualized and were trackable in vivo. Acute endothelial rejection in 1 rabbit was evidenced by detectable DIR positive cells within the anterior chamber. DIR imaging allowed for detailed imaging of the transplanted corneal endothelium, and enabled non-invasive observation of the corneal endothelial morphology following transplantation.

\section{Introduction}

The cornea is the most powerful lens system in the eye, meaning subtle alterations in its clarity and shape have significant impacts on the quality of vision ${ }^{1,2}$. Corneal clarity is reliant on the function of the corneal endothelium, a monolayer of non-replicative cells critical for the regulation of corneal hydration. If the corneal endothelial function is impaired, corneal oedema occurs, followed by scarring and vascularization if left untreated ${ }^{3}$. At present, endothelial failure can only be reversed by allogenic corneal transplantation and endothelial dysfunction is the leading indication for transplantation in the developed world ${ }^{4}$.

As there is a global shortage of donor corneal graft tissue, methods to improve transplant success and longevity, as well as to eliminate the 'one-donor for one-recipient' paradigm are greatly needed ${ }^{4}$. All strategies are reliant on replacing the corneal endothelial cells (CECs), either by transplantation or regenerative strategies ${ }^{5}$. Corneal endothelial function is dependent upon adequate corneal endothelial cell density (ECD), with corneal oedema occurring when the ECD drops below $400-600 \mathrm{cells} / \mathrm{mm}^{2}$. This also applies to donor corneas following transplantation ${ }^{6}$, and is, therefore, highly likely to be also the case in endothelial cell repopulation following cell injection or regeneration ${ }^{5,7}$.

After corneal transplantation, a rapid decline in corneal ECD is observed following surgery ${ }^{8,9}$. We have previously shown that iatrogenic cell loss occurs during the preparation ${ }^{10}$, storage ${ }^{11}$, insertion and unfolding of endothelial grafts ${ }^{12}$. In addition, factors such as air tamponade ${ }^{13,14}$, post-operative inflammation and co-morbidities such as glaucoma ${ }^{15,16}$, are likely to affect early endothelial cell loss. 
Immediately after transplantation, areas of dead or denuded endothelial cells are present ${ }^{12}$. A process of cell migration and restoration of an intact monolayer must occur in the early post-operative period in order for corneal clarity and thickness to be restored to normal levels ${ }^{17}$.

Monitoring of endothelial cells can either be performed directly by imaging the cells ${ }^{17}$ or indirectly by using measurements of endothelial function: central corneal thickness (CCT) and transparency ${ }^{18}$. Presently, endothelial cells are imaged in vivo in the clinical setting using specular or confocal microscopy ${ }^{19}$, although newer modalities, based on optical coherence tomography (OCT) imaging, are being developed ${ }^{20}$. Typically, only a small number of cells, between 30 to 100 , are imaged at high magnification ${ }^{21}$. Image quality can be poor following endothelial keratoplasty (EK), especially with noncontact methods ${ }^{22}$. Additionally, poor image acquisition from off-axis portions of the cornea and the lack of automated methods to stitch multiple images together, mean these methods do not allow for global assessment of the entire graft ${ }^{23}$. Consequently, tracking small areas of interest over time can be very difficult ${ }^{24}$. In both specular and confocal microscopy, image quality is reliant on a clear corneal stroma, meaning images obtained through oedematous or scarred corneas are often of poor quality. The presence of an intra-cameral gas bubble can also impair image quality (personal observations). As such, it is often not possible to use these methods in the early post-operative period following EK, nor is it possible to use these methods to examine endothelial healing following wounding studies.

In our previous studies, in vivo endothelial wound healing was assessed using repeated intracameral trypan blue injection ${ }^{17}$. Whilst this was a reliable and reproducible technique, it is an invasive procedure that carries the risk of damaging healing endothelial cells or introducing infections ${ }^{25}$. We were reluctant to utilize this method following EK or Descemet's membrane transplantation for fear of dislodging the recently transplanted material. An additional limitation of trypan blue technique is that it only allowed global imaging of endothelial wound healing. This meant animals had to be sacrificed at staggered time intervals in order to perform histological evaluation of wound healing at a cellular level.

We previously developed and validated a method to image endothelial cells, in vivo, using Calcein AM; a green-fluorescent viability stain ${ }^{12}$. Whilst capable of global and cell-level imaging in the immediate postoperative period, our in vitro experiments showed that Calcein AM was rapidly cleared from endothelial cells meaning a useful fluorescence signal had been extinguished by 24 hours post incubation. As such, it is not useful for longer-term tracking of endothelial cells in vivo. The fate of the transplanted cells can only truly be determined if methods to quantify endothelial cell death immediately post-operatively can be combined with techniques to track endothelial cells through early post-operative phase, which we define as one month based on previous reports ${ }^{26,27}$.

In this study, we describe a technique for labelling and tracking endothelial cells in vivo, both globally across the entire cornea and at an individual cell level. We compared these in vivo images to immunohistology following animal sacrifice. We show this technique is suitable for tracking both native and 
transplanted endothelial cells and can be used in wound healing studies as well as xenotransplantation of human EK grafts into rabbits.

\section{Results}

\section{Optimizing DIR concentration in vitro}

Average DIR fluorescence increased with concentration in a linear-quadratic fashion. Comparison of fluorescence levels at different doses revealed a significant difference between cells stained with $50 \mu \mathrm{g} / \mathrm{ml}$ and $100 \mu \mathrm{g} / \mathrm{ml}$ (ANOVA, corrected $\mathrm{p}<0.0001$ ) but not between $100 \mu \mathrm{g} / \mathrm{ml}$ and $200 \mu \mathrm{g} / \mathrm{ml}(\mathrm{p}=0.78)$ (Figure 1A). Based on these findings a dose of $100 \mu \mathrm{g} / \mathrm{ml}$ was chosen for in vitro subsequent labelling. A dose of $200 \mu \mathrm{g} / \mathrm{ml}$ was chosen for intracameral injection as it was not possible to remove all aqueous prior to DIR injection meaning additional dilution occurred in the anterior chamber. No significant differences were found on toxicity assessment between DIR at any concentration and matched controls (Figure 1B). Wound healing kinetics were similar between control and treated groups (Figure 2). There was no significant difference in wound area remaining at any time point.

\section{In vivo labelling of rabbit endothelial cells}

Direct injection of DIR diluted in BSS was successful in labelling rabbit endothelial cells. No adverse effects were observed with no significant inflammation observed following the injection. Slit lamp microscopy, OCT and infra-red imaging on day 1 showed no change in corneal clarity (Figure 3A), with DIR fluorescence showing observable cell-to-cell variation (Figure 3B). There was no increase in corneal thickness (Figure 3C). At day 28, the cornea remains clear (Figure 3D), where labelled cells were still visible, but with some reduction in observable fluorescence (Figure 3E). DIR uptake was prominent in the iris, meaning that pupil dilation was necessary to reduce background fluorescence in subsequent examinations. Background fluorescence was further reduced by imaging using the higher magnification lenses, as these reduced the depth of focus, placing the iris beyond the plane that could be imaged (Figure 3E). Throughout the study period, the corneal thickness remained normal (Figure 3F).

\section{In vivo corneal wounding: Comparison between in vivo DIR imaging, confocal microscopy and immunohistology.}

At a microscopic level, there was good agreement between cellular images acquired using in vivo DIR imaging and those obtained after immunofluorescence staining (Figure 4). During early wound healing (day 3), elongated cells without clear cell borders were seen using both imaging techniques (Figure 4A). There was no difference in cell circularity measured from DIR and immunofluorescence images, with an average value of $0.51 \pm 0.14$ for histology derived circularity and $0.58 \pm 0.12$ for DIR imaging $(p=0.34)$. After wound closure, a confluent monolayer of cells with broad cell borders was seen with in vivo DIR imaging. This corresponded to confluent cells with incomplete tight junction formation, as assessed by ZO-1 immunofluorescence staining (Figure 4B). After initial wound closure, a continuing maturation of central endothelial cells was observed, mirroring the findings in unstained rabbit endothelial cells. As time 
progressed cell borders became narrower and cells became more circular (Figure 4C). This corresponded with the expression patterns of ZO-1 staining at day 3 (Figure 4D), day 5 (Figure 4E), as well as day 28 where a continuous ring of ZO-1 staining were detected on immunofluoresnce (Figure 4F). Conventional in vivo confocal images could not be acquired at day 3 (Figure 4G) and day 5 (Figure 4H). However, from day 14 onward, useful confocal images could be obtained. Analysis at day 28 showed no difference in cell circularity ( 0.71 vs $0.74 p=0.87)$ or the number of hexagonal cells as assessed by histology and DIR imaging. (50.9\% (histology) vs $49.1 \%$ (Spectralis), $p=0.9$ )

\section{In vivo corneal wounding: Comparison between in vivo DIR imaging and trypan blue staining}

Good agreement was seen between macroscopic, non-invasive DIR imaging and intracameral trypan blue staining, both at the time of wound creation (Figure 5A and 5B) and at day 3 after wounding (Figure 5C and 5D). More importantly, these images can be acquired through corneas that were thicken or irregular in shape (Figure 5E). Bland-Altman plots were produced to compare the area and circularity of wounds (Figure 6). Good agreement was seen between both methods, with less than $2 \%$ difference in area seen in any sample (Figure $5 \mathrm{~A}$ ). Circularity was within $4 \%$ for all samples (Figure $5 \mathrm{~B}$ )

(E) OCT image of the cornea imaged at day 3 post wounding confirms DIR images are successfully acquired through thickened and irregular corneas.

\section{In vivo tracking of migrating endothelial cells following endothelial wounding in the rabbit}

In keeping with our previously published results ${ }^{17}$, healing of an $8 \mathrm{~mm}$ endothelial scrape wound occurred by day 5 , with a return to pre-wound CCT by day 14 in all cases (Figure 7). These findings suggest that DIR staining does not have a deleterious impact on endothelial healing or migration in vivo.

\section{Labelling of ex vivo human corneas}

Double staining using Calcein AM and DIR successfully labelled donor human corneas within an ex vivo experimental setup pre-op (Figure 8A and 8B), as well as immediately post operatively (Figure $8 \mathrm{C}$ and 8D). Initially, staining of DIR appeared variable, with adjacent areas of the corneal endothelium showing markedly different degrees of fluorescence (Figure 8B and 8D). When compared with Calcein AM staining, it revealed that DIR staining was not able differentiate between living and dead endothelial cells that remained attached to the Descemet's Membrane (Figure 8E and 8F). As predicted by our in vitro assessment ${ }^{12}$, Calcein AM fluorescence diminished quickly, and no useful images could be obtained at 24 hrs post implantation (Figure 8G). Interestingly, DIR fluorescence tended to become more even by 24 hours post graft insertion, meaning large areas of cell loss could be seen in clear contrast to areas covered by cells (Figure $8 \mathrm{H}$ ).

\section{Tracking transplanted human endothelial cells in vivo}

EK grafts labelled with DIR maintained strong fluorescence for the entire study period ( 28 days). Cell migration could be seen as endothelial damage associated with tissue insertion healed (Figure 9A; red 
arrows). Transfer of fluorescence to neighboring rabbit endothelial cells was not observed and the patterns of fluorescence seen in vivo closely matched those observed histologically (Figure 9B-9E). Areas of endothelial migration off the donor and onto the host DM were seen in DMEK cases (Figure 9B and 9C) but not in DSAEK.

In one animal that had DMEK, clinical features of rejection (corneal oedema, ciliary injection) was observed at day 28 (Figure 10). Infrared imaging was able to detect a central loss of fluorescence associated with the onset of circulating, DIR positive, cells in the anterior chamber (Video S1). An anterior chamber tap confirmed the presence of cellular material but the yield was too low to confirm cell origin or DIR positivity using FACS/immunofluorescence.

\section{High resolution cell imaging}

By attaching the Rostock module to the Spectralis HRA, we were able to acquire detailed, high-resolution in vivo images. This allowed us to observe subtle biological processed at a cellular level including nuclear fragmentation and cell detachment in both rabbits (Figure 11A and 11B) and human (Figure 11C) corneal endothelium; podocyte extension from neighboring cells (Figure 11D); nuclei in anaphase (Figure 11E) and daughter cell formation (Figure 11F).

\section{Discussion}

Tracking of CECs in vivo is not widely performed to date due to a lack of suitable imaging techniques and paucity of data on labelling efficiency, duration of fluorescence, general toxicity, and effects of labelling on functionality of transplanted tissue ${ }^{28}$. The aim of this study was to determine whether NIR dye, and specifically for this study, DIR, could be used to label and track CECs, in vivo, without impacting both cell viability and cellular function. We began by assessing the effect of DIR staining on wound healing, cell migration and cell viability, in vitro, using cultured primary human CEC's. At the concentrations required for in vivo imaging, DIR stained CECs migrated at the same rate as unlabeled CECs. At 24 hrs postlabelling, there was no significant difference in cell death, as assessed by the Calcein AM/Ethidium Homodimer assay, between labelled and unlabeled cells. Taken together, these results suggest that DIR was a viable candidate for use as a tracker in CECs. We subsequently assessed whether use of DIR had any deleterious effects in vivo. A single intracameral DIR injection was sufficient to label these cells and was not associated with any apparent inflammation or acute disturbance of corneal endothelial function. We subsequently used DIR imaging to track the endothelial cells on EK transplants from human tissue. We found it was possible to obtain high quality images of human endothelial cells within the anterior chamber following EK. To our knowledge, this is the first-time biological processes such as healing of iatrogenic wounds and migration of endothelial cells from donor to host have been observed in vivo.

At the macroscopic level, we found DIR imaging to be comparable to repeated trypan blue injection when assessing endothelial wound healing following standard injury; a model commonly used to assess pharmacological therapies aimed at enhancing endothelial regeneration ${ }^{29-31}$. High magnification 
assessment of the healing edge of the wound showed that comparable morphological information could be obtained from in vivo imaging as compared to immuno-histology following animal sacrifice. A temporal relationship between closure of endothelial wounds, as observed by DIR imaging, and restoration of corneal clarity/CCT was also seen in all cases. In vivo wound healing kinetics were similar to those we previously reported in unlabeled cells ${ }^{17}$. Images could be acquired through opaque/edematous corneas, something not possible with conventional confocal imaging. Combined, these findings suggest that DIR staining has no significant effect on endothelial function or healing and allows continuous, in vivo, assessment at all time points post wounding.

Assessment of endothelial transplants using DIR tracker showed there was no diffusion of DIR across cell membranes from adjacent endothelial cells, meaning that staining of rabbit endothelial cells did not occur following xenotransplantation with human DMEK grafts (Figure 8). This allowed for a clear distinction between transplanted human endothelial cells and native rabbit endothelial cells, even if cells had migrated on or off the transplant itself. Until now, detailed assessment of wound healing at different time points has relied either on repeated surgical interventions, in the case of trypan blue injection ${ }^{17}$, or on the sacrifice of animals at multiple time points ${ }^{32}$. Our assessments showed that a DIR fluorescence signal, allowing for high quality image acquisition, was retained for at least 28 days and in subsequent experimentation we have found fluorescence to be retained up to 60 days (data not shown).

With high resolution imaging, biological processes typically observed on histological examination could be seen in vivo; including cell spreading, apoptosis and cell division. In one animal, a reduction in corneal clarity, together with a development of corneal edema and an increase in corneal thickness occurred between days 21 and 28. DIR imaging showed cells had become rounded, detached from the graft and were freely floating in the aqueous. The cells remaining attached to DM displayed features consistent with an acute insult ${ }^{20}$. In the setting of xenotransplantation, these features were consistent with acute graft rejection ${ }^{33,34}$. In keeping with this, small DIR positive cells, consistent with previous reports of inflammatory cells, were observed interspersed with the endothelial cells were seen in both in the rabbit endothelial monolayer and transplanted human endothelial cells ${ }^{35}$.

It is imperative to determine the success of new endothelial repair strategies, including cell injection and tissue-engineered EK grafts ${ }^{32}$, prior to their clinical adoption. We have previously shown that rabbit endothelial cells are capable of migrating over bare DM, either native or following transplantation, with subsequent restoration of corneal clarity and thickness to pre-transplantation levels ${ }^{17}$. As such, it is important to be certain that any restoration of function is attributable to the transplanted cells and not a consequence of cellular regeneration in models where in vivo endothelial replication occurs ${ }^{36}$. In order to achieve this, some authors resort to either short term observation without complete restoration of corneal clarity to draw conclusions about transplant success ${ }^{36}$. Others resort to the use primate models for corneal endothelial research, as their native endothelial cells are thought to have a limited regenerative potential ${ }^{37}$. In many parts of the world a concerted effort to limit the use of primate models is being introduced (https://www.nc3rs.org.uk/welfare-non-human-primates). DIR staining may allow successful 
use of non-primate and non-animal models for endothelial tracking by allowing researchers to determine whether corneal recovery has occurred due to the persistence of transplanted cells or by their replacement, without resorting to animal sacrifice.

In addition, to the ability to track endothelial cells, this technique may also be a valuable tool to study biological processes such as graft rejection, which remains a leading cause of transplant failure. Currently, models of rejection rely on indirect measures of endothelial function, and it is not always possible to separate rejection from other forms of endothelial trauma ${ }^{18,38}$. Methods of imaging inflammatory cell changes that proceed acute rejection in vivo will allow a greater understanding of the mechanisms at play ${ }^{39}$. Being able to assess the degree of cell death occurring during a rejection episode will provide information on the likelihood of recovery following rejection and effectiveness of antirejection strategies.

The use of NIR markers for tracking has several advantages. These tracers benefit from very low autofluorescence at NIR wavelengths and has excellent tissue penetration. This combination gives a high signal-to-noise ratio and allows non-invasive tracking of cells through the skin in organs such as the spleen or heart ${ }^{40}$. NIR tracers have recently been used in surgical trials to aid tumor clearance ${ }^{41}$ and in clinical ophthalmic trials to assess cell loss in patients with early glaucoma ${ }^{42}$. Using an $800 \mathrm{~nm}$ band pass, sufficient emitted light is captured for high quality imaging, and this is possible even through opaque corneas with significant edema. The NIR dye, indocyanine green (ICG), has been used in ophthalmology for over 5 decades. This means that dedicated equipment for imaging of NIR tracers in the eye are widely available in clinical and research settings. Our imaging protocol uses clinical equipment, designed for ICG imaging, with minimal adjustments.

Given that DIR staining is a simple, non-toxic, one step process, it is possible that this method could be applied to graft tissue destined for human use if a good manufacturing practice form of the dye were made available. For organ cultured tissue, the point at which graft tissue is transferred to dextran containing media may provide an ideal opportunity for this ${ }^{43}$. More detailed information about endothelial cell behavior following transplantation will improve our understanding of phenomena such as accelerated cell loss in high risk grafts and may lead to targeted therapeutics and improved graft survival. Our study showed that DIR fluorescence was initially quite heterogenous. This meant multiplexing with the viability dye, Calcein AM, was needed to assess immediate post-op graft viability. Fluorescence peaked at $24 \mathrm{hrs}$ post transplantation making imaging easier, although some heterogeneity remained meaning appropriately exposing the images was challenging. It may be possible to improve the use of DIR by combining several images taken at different sensitivities; high dynamic range imaging ${ }^{44}$. It is possible that DIR may also be useful to track other cells used in corneal regenerative therapy such as limbal epithelial ${ }^{28}$ or stromal stem cells ${ }^{45}$.

We believe that DIR imaging provides a powerful new tool for studying CECs in vivo. Its use in a research setting should ultimately lead to a reduction in the number of research animals needed and there is a potential for easy adoption in the clinical setting. 


\section{Methods}

\section{Study Tissue}

Experimentation was conducted in accordance with the tenets of the Declaration of Helsinki. Human corneo-scleral buttons with consent for research use were obtained from Miracles in Sight (Winson Salem, North Carolina, USA). Tissue preparation and transplantation was performed by a single surgeon (MB). Transplant grade tissue with endothelial cell counts of $>2200$ cells $/ \mathrm{mm}^{2}$ and a storage time $<14$ days in Optisol-GS (Bausch \& Lomb Rochester, New York) was used for all experimentation.

\section{Research Animals}

Animals procured for this study were treated in accordance with the ARVO Statement for Use of Animal in Ophthalmic and Visual Research as well as the ARRIVE guidelines ${ }^{46}$, and all experiments involving these animals were approved by the Singhealth Institutional Animal Care and Use Committee (IACUC; 2016/SHS/1212), Singapore. Interventions were performed in New Zealand white rabbits aged 16-20 weeks, weighing between $3 \mathrm{~kg}$ to $3.5 \mathrm{~kg}$. All surgical procedures and clinical evaluations were performed in a single eye of each rabbit under general anesthesia from intra-muscular xylazine $5 \mathrm{mg} / \mathrm{kg}$ (Troy Laboratories, Smithfield, Australia) and ketamine 40mg/kg (Parnell Laboratories, Alexandria, Australia) with additional topical anesthesia (lidocaine $1 \%$, Bausch and Lomb, Rochester) as previously described ${ }^{17}$. Animals underwent phacoemulsification at least 1 week prior to endothelial keratoplasty to deepen the anterior chamber. Vancomycin was used in the irrigating fluid $(20 \mu \mathrm{g} / \mathrm{ml})$ for surgical procedures and rabbits received subconjunctival dexamethasone $(1 \mathrm{mg})$ and gentamcin $(0.4 \mathrm{mg})$ at the end of all surgical procedures.

Prior to EK, rabbits were injected with intravenous unfractionated heparin (1000 units in $1 \mathrm{ml}$ of normal saline). Heparin was also added to the balanced salt solution used to irrigate the eye at a concentration of 50 units $/ \mathrm{ml}$. For all experiments, rabbits were examined daily for the first 5 days and then on days 7 , 14, 21, and 28. Examinations consisted of slit lamp biomicroscopy, tonometry, optical coherence tomography, confocal fluorescence imaging using the Heidelbrg Spectralis (Heidelberg Engineering $\mathrm{GmbH}$, Heidelberg, Germany) and conventional corneal confocal imaging using the Heidelberg HRT-II with the Rostock module.

\section{Optimizing DIR concentration in vivo}

A stock solution of 1,1'-dioctadecyl-3,3,3',3'-tetramethylindotricarbocyanine iodide (DIR) dye was made by dissolving $10 \mathrm{mg}$ of DIR powder in $1 \mathrm{ml}$ of pure ethanol (ThermoFisher Scientific, USA).

Primary cultured human CECs were seeded at a concentration of $2 \times 10^{5}$ cells $/ \mathrm{cm}^{2}$ in a Greiner 48-well flat bottom polystyrol microplate (Greiner, Frickenhausen, Germany), pre-coated with FNC coating mix. Cells were incubated with DIR diluted in M5 media ${ }^{47}$ at a concentration of $10-200 \mu \mathrm{g} / \mathrm{ml}$ overnight at $37^{\circ} \mathrm{C}$. The 
following day, cells were washed thrice in fresh culture media and imaged using the Spectalis at a standardized sensitivity of 100. Images were exported to ImageJ (National Institute of Health, Bethesda, USA) and converted to 8-bit. Mean grey values were measured in three separate regions of each plate and average fluorescence for each dose calculated.

\section{Toxicity of DIR in vitro}

DIR-labelled endothelial cells and controls were cultured for a further $48 \mathrm{hrs} \mathrm{in} \mathrm{fresh} \mathrm{media.} \mathrm{Calcein}$ AM/ethidium homodimer/Hoechst were added to the cultured cells to achieve final concentrations of $2 \mu \mathrm{M}, 4 \mu \mathrm{M}$ and $10 \mu \mathrm{M}$ respectively. The plate was gently agitated, incubated at $37^{\circ} \mathrm{C}$ for 30 minutes and imaged on the fluorescence microscope. The viable ECD was calculated and compared between control and treated cells. A positive control was performed by using the same viability assessment in cells incubated with $70 \%$ ethanol for 1 minute prior to assessment.

\section{Effect of DIR on endothelial cell migration}

As the primary aim of DIR tracking was to examine cell migration, a scratch wound assay was performed. Primary cultured HCEC's from the same donors were seeded onto different wells within the same plate. After reaching confluence, cells were cultured in M5 culture media with DIR $10 \mu \mathrm{g} / \mathrm{ml}$ or M5 media alone (controls). Each well received a single scratch using the tip of a $10 \mu$ pipette. Plates were placed within the incuCyte imaging incubator (Sartorius, Essen Bioscience, Ann Arbor, Michigan, USA). The incuCyte is a multiplex imaging system that is incorporated into an incubator, allowing continuous imaging in multiple wells without alterations in temperature or atmosphere. Image registration to define the scratch area was performed and serial phase-contrast images were taken every hour for $48 \mathrm{hrs}$. The wound area was measured at $\mathrm{T}=12 \mathrm{hrs}$ and expressed as a percentage of that measured at $\mathrm{T}=0 \mathrm{hrs}$.

\section{Labelling of rabbit endothelial cells in vivo}

A single, self-sealing paracentesis was created with a $1 \mathrm{~mm}$ diamond blade. DIR, diluted in BSS at a concentration of $200 \mu \mathrm{g} / \mathrm{ml}$ was drawn into an insulin syringe and the needle inserted into the anterior chamber at the limbus, taking care to tunnel the entry for at least $1 \mathrm{~mm}$. The posterior lip of the paracentesis was depressed causing expression of the aqueous after which the DIR was slowly injected thus performing a DIR/aqueous exchange. The rabbits were kept with the treated eye facing down until the anaesthesia had worn off (approximately 45 mins).

\section{Imaging of endothelial cells post-operatively}

Imaging was performed using the Spectralis HRT-II set in the ICG mode. In the ICG mode, the Spectralis utilizes a $790 \mathrm{~nm}$ diode excitation laser and $800 \mathrm{~nm}$ long-pass filter. Images were captured at varying levels of magnification by attaching microscope objective lenses (Nikon, Japan) or the Rostock module to the standard fundus imaging lens by using custom built collars, as previously described. Use of multiple lenses allowed both macroscopic and individual cell level imaging. 


\section{Comparison of DIR imaging and established methods in a wound healing model}

Two weeks following DIR injection, 4 Rabbits had $8 \mathrm{~mm}$ endothelial scrape wounds as described in our previous work ${ }^{17}$. One rabbit was sacrificed at day 3 , one at day 5 and the remaining two animals were sacrificed at day 28 . The healing edge of cells was imaged by high magnification in vivo DIR imaging and compared to conventional in vivo confocal microscopy and immunohistology.

Following sacrifice, corneas were harvested, washed in BSS and fixed in 1\% paraformaldehyde at room temperature for 5 minutes. Samples were permeabilized in Triton-X $1 \%$ for 5 minutes and then blocked using and 2 step process, first in 10\% goat serum for 45 minutes followed by $3 \%$ BSA for 45 mins. Samples were incubated with primary antibody to ZO-1 (1:300, Thermofischer) or Human Nuclear Antigen (1:100, Merck-Millipore) at $4^{\circ} \mathrm{C}$ for 24 hours in a solution composed of phosphate buffered saline (PBS) with $0.1 \%$ Triton-X and $1 \%$ BSA. Samples were washed $x 3$ with PBS, each for 10 minutes, and then incubated with secondary antibody, Hoechst (1:1000) and phalloidin (1:500), as required, overnight at $4^{\circ} \mathrm{C}$. To assess specificity of the immunostaining, corneas were processed without primary antibody. Specimens were washed $x 3$ in PBS, re-fixed in 3\% PFA, washed again and flat mounted in ProLong gold. Individual cells were manually outlined and cell metrics compared between in vivo images and flatmounted, immunofluorescence images (stained with phalloidin, Hoechst and ZO-1). At day 3, when cells were still migrating, a comparison between cell circularity was made (a minimum of 100 cells were measured in each group). At day 28 when a stable monolayer was present, a comparison between cell circularity and the percentage of hexagon cells was made (neighbor analysis function in the BioVoxxel toolbox for ImageJ).

\section{Labelling of ex vivo human corneas}

Human corneas were labelled prior to tissue preparation for Descemet Membrane Endothelial Keratoplasty (DMEK) and following mircokeratome cutting for Descemet Stripping Automated Endothelial Keratoplasty (DSAEK). The procedure was optimized in a series of initial experiments. To limit the amount of dye required and prevent corneal swelling, the corneas were kept within the Optisol-filled viewing chamber. Optisol was aspirated from the corneal cup and the endothelial surface further dried with cellulose sponges. DIR $(100 \mu \mathrm{g} / \mathrm{ml})$, diluted in M5 medium, was pipetted onto the endothelial surface until the entire endothelium and trabecular meshwork were covered. The epithelial surface remained suspended by the pillars of the viewing chamber and in contact with the Optisol, with the scleral rim acting as barrier to prevent the two media for mixing. The lid for the viewing chamber was replaced loosely and the corneas were incubated over night at $37^{\circ} \mathrm{C}$. For corneas in which a comparison between DIR and Calcein AM fluorescence was performed, corneas were rinsed $\mathrm{x} 3$ in fresh M5 media and the incubated with Calcein AM $1.67 \mu \mathrm{M}$ in BSS for 30 mins prior to surgery.

Tissue was prepared and implanted using our previously described techniques for DMEK $(n=4)^{12,48}$ and DSAEK $(n=10)^{49}$ and imaging for Calcein AM and DIR fluorescence performed alongside the standard examinations, following the review schedule outlined above. 
Six rabbits underwent DSAEK with DIR labelled tissue. Two weeks after implantation, $4.5 \mathrm{~mm}$ endothelial wounds were created. Endothelial wounds were delineated with trypan blue, as previously published.

Trypan blue and DIR images were exported to ImageJ and the wound outlined using the polygon selection tool. This was converted to a smoothed spline and measurements of wound area and circularity were taken. The measurements for imaging technique were compared using a Bland-Altman plots.

\section{Abbreviations}

CECs: corneal endothelial cells; ECD: endothelial cell density; CCT: central corneal thickness; OCT: optical coherence tomography; EK: endothelial keratoplasty; NIR: near-infrared; DIR: 1,1'-dioctadecyl-3,3,3',3'tetramethylindotricarbocyanine iodide; DM: Descemet's Membrane; DMEK: Descemet Membrane Endothelial Keratoplasty; DSAEK: Descemet Stripping Automated Endothelial Keratoplasty; ICG: indocyanine green.

\section{Declarations}

\section{Acknowledgments}

We thank Lion Eye Institute for Transplant and Research and Miracles in Sights for their assistance with procurement of all the research-grade donor corneas used in this study. This research is supported by the Singapore Ministry of Health's National Medical Research Council under its Clinician Scientist Award (CSA) (MOH-000197). The funding bodies had no role in study design, data collection, analysis, decision to publish, or preparation of the manuscript.

\section{Author contributions}

MB and JSM conceptualized and designed the study; MB and GSP supervised the research; SJL and KA carried out the in vitro characterization studies and contributed to data analysis; MB, HPA, and CNL were involved in the animal studies. MB coordinated the study and together with GSP and JSM prepared the manuscript.

\section{Additional information}

\section{Competing Interests}

The authors have declared that no competing interest exists.

\section{References}

1 Laing, R. A., Sanstrom, M. M., Berrospi, A. R. \& Leibowitz, H. M. Changes in the corneal endothelium as a function of age. Exp Eye Res 22, 587-594 (1976). 
2 Edelhauser, $\mathrm{H}$. F. The resiliency of the corneal endothelium to refractive and intraocular surgery. Cornea 19, 263-273, doi:10.1097/00003226-200005000-00002 (2000).

3 Tuft, S. J. \& Coster, D. J. The corneal endothelium. Eye (Lond) 4 ( Pt 3), 389-424, doi:10.1038/eye.1990.53 (1990).

4 Tan, D. T., Dart, J. K., Holland, E. J. \& Kinoshita, S. Corneal transplantation. Lancet 379, 1749-1761, doi:10.1016/S0140-6736(12)60437-1 (2012).

5 Kinoshita, S. et al. Injection of Cultured Cells with a ROCK Inhibitor for Bullous Keratopathy. N Engl J Med 378, 995-1003, doi:10.1056/NEJMoa1712770 (2018).

6 Bourne, W. M. Cellular changes in transplanted human corneas. Cornea 20, 560-569, doi:10.1097/00003226-200108000-00002 (2001).

7 Fuest, M., Yam, G. H., Peh, G. S. \& Mehta, J. S. Advances in corneal cell therapy. Regen Med 11, 601-615, doi:10.2217/rme-2016-0054 (2016).

8 Gauthier, A. S. et al. Very early endothelial cell loss after penetrating keratoplasty with organcultured corneas. Br J Ophthalmol 101, 1113-1118, doi:10.1136/bjophthalmol-2016-309615 (2017).

9 Monnereau, C. et al. Multicenter study of descemet membrane endothelial keratoplasty: first case series of 18 surgeons. JAMA Ophthalmo/ 132, 1192-1198, doi:10.1001/jamaophthalmol.2014.1710 (2014).

10 Bhogal, M., Balda, M. S., Matter, K. \& Allan, B. D. Global cell-by-cell evaluation of endothelial viability after two methods of graft preparation in Descemet membrane endothelial keratoplasty. $\mathrm{Br} J$ Ophthalmol 100, 572-578, doi:10.1136/bjophthalmol-2015-307534 (2016).

11 Bhogal, M., Matter, K., Balda, M. S. \& Allan, B. D. Organ culture storage of pre-prepared corneal donor material for Descemet's membrane endothelial keratoplasty. Br J Ophthalmol 100, 1576-1583, doi:10.1136/bjophthalmol-2016-308855 (2016).

12 Bhogal, M. et al. Real-time assessment of corneal endothelial cell damage following graft preparation and donor insertion for DMEK. PLoS One 12, e0184824, doi:10.1371/journal.pone.0184824 (2017).

13 Feng, M. T., Price, M. O., Miller, J. M. \& Price, F. W., Jr. Air reinjection and endothelial cell density in Descemet membrane endothelial keratoplasty: five-year follow-up. J Cataract Refract Surg 40, 1116-1121, doi:10.1016/j.jcrs.2014.04.023 (2014).

14 Landry, H. et al. Corneal endothelial toxicity of air and SF6. Invest Ophthalmol Vis Sci 52, 22792286, doi:10.1167/iovs.10-6187 (2011). 
15 Mitry, D. et al. Descemet stripping automated endothelial keratoplasty after failed penetrating keratoplasty: survival, rejection risk, and visual outcome. JAMA Ophthalmo/ 132, 742-749, doi:10.1001/jamaophthalmol.2014.352 (2014).

16 Maurino, V. \& Aiello, F. Glaucoma risks in advanced corneal surgery. Prog Brain Res 221, 271-295, doi:10.1016/bs.pbr.2015.06.009 (2015).

17 Bhogal, M., Lwin, C. N., Seah, X. Y., Peh, G. \& Mehta, J. S. Allogeneic Descemet's Membrane Transplantation Enhances Corneal Endothelial Monolayer Formation and Restores Functional Integrity Following Descemet's Stripping. Invest Ophthalmol Vis Sci 58, 4249-4260, doi:10.1167/iovs.17-22106 (2017).

18 Liu, Y. C., Lwin, N. C., Chan, N. S. \& Mehta, J. S. Use of anterior segment optical coherence tomography to predict corneal graft rejection in small animal models. Invest Ophthalmol Vis Sci 55, 67366741, doi:10.1167/iovs.14-14475 (2014).

19 Rio-Cristobal, A. \& Martin, R. Corneal assessment technologies: current status. Surv Ophthalmol 59, 599-614, doi:10.1016/j.survophthal.2014.05.001 (2014).

20 Ang, M. et al. Evaluation of a Micro-Optical Coherence Tomography for the Corneal Endothelium in an Animal Model. Sci Rep 6, 29769, doi:10.1038/srep29769 (2016).

21 McCarey, B. E., Edelhauser, H. F. \& Lynn, M. J. Review of corneal endothelial specular microscopy for FDA clinical trials of refractive procedures, surgical devices, and new intraocular drugs and solutions. Cornea 27, 1-16, doi:10.1097/IC0.0b013e31815892da (2008).

22 Raecker, M. E., McLaren, J. W., Kittleson, K. M. \& Patel, S. V. Endothelial image quality after descemet stripping with endothelial keratoplasty: a comparison of three microscopy techniques. Eye Contact Lens 37, 6-10, doi:10.1097/ICL.0b013e318203dc19 (2011).

23 Tanaka, H. et al. Panoramic view of human corneal endothelial cell layer observed by a prototype slit-scanning wide-field contact specular microscope. Br J Ophthalmol 101, 655-659, doi:10.1136/bjophthalmol-2016-308893 (2017).

24 Gasser, L., Daniel, M., Reinhard, T. \& Bohringer, D. Long-term tracking of the central corneal endothelial mosaic. PLoS One 9, e88603, doi:10.1371/journal.pone.0088603 (2014).

25 Ong, A. P. et al. Post-surgical versus post-intravitreal injection endophthalmitis: changing patterns in causative flora. Clin Exp Ophthalmol 47, 57-62, doi:10.1111/ceo.13345 (2019).

26 Bahn, C. F. et al. Penetrating keratoplasty in the cat. A clinically applicable model. Ophthalmology 89, 687-699, doi:10.1016/s0161-6420(82)34750-8 (1982). 
27 Haydari, M. N. et al. A short-term in vivo experimental model for Fuchs endothelial corneal dystrophy. Invest Ophthalmol Vis Sci 53, 6343-6354, doi:10.1167/iovs.12-9708 (2012).

28 Genicio, N., Gallo Paramo, J. \& Shortt, A. J. Quantum dot labeling and tracking of cultured limbal epithelial cell transplants in vitro. Invest Ophthalmol Vis Sci 56, 3051-3059, doi:10.1167/iovs.14-15973 (2015).

29 Hoppenreijs, V. P., Pels, E., Vrensen, G. F. \& Treffers, W. F. Corneal endothelium and growth factors. Surv Ophthalmol 41, 155-164, doi:10.1016/s0039-6257(96)80005-1 (1996).

30 Nakano, Y. et al. Connexin43 knockdown accelerates wound healing but inhibits mesenchymal transition after corneal endothelial injury in vivo. Invest Ophthalmol Vis Sci 49, 93-104, doi:10.1167/iovs.07-0255 (2008).

31 Meekins, L. C. et al. Corneal Endothelial Cell Migration and Proliferation Enhanced by Rho Kinase (ROCK) Inhibitors in In Vitro and In Vivo Models. Invest Ophthalmol Vis Sci 57, 6731-6738, doi:10.1167/iovs.16-20414 (2016).

32 Koizumi, N., Okumura, N. \& Kinoshita, S. Development of new therapeutic modalities for corneal endothelial disease focused on the proliferation of corneal endothelial cells using animal models. Experimental Eye Research 95, 60-67, doi:10.1016/j.exer.2011.10.014 (2012).

33 Qian, Y. \& Dana, M. R. Molecular mechanisms of immunity in corneal allotransplantation and xenotransplantation. Expert Rev Mol Med 3, 1-21, doi:10.1017/S1462399401003246 (2001).

34 Ross, J. R., Howell, D. N. \& Sanfilippo, F. P. Characteristics of corneal xenograft rejection in a discordant species combination. Invest Ophthalmol Vis Sci 34, 2469-2476 (1993).

35 Koudouna, E. et al. Immune Cells on the Corneal Endothelium of an Allogeneic Corneal Transplantation Rabbit Model. Invest Ophthalmol Vis Sci 58, 242-251, doi:10.1167/iovs.16-20019 (2017).

36 Hitani, K. et al. Transplantation of a sheet of human corneal endothelial cell in a rabbit model. Mol Vis 14, 1-9 (2008).

37 Koizumi, N. et al. Cultivated corneal endothelial cell sheet transplantation in a primate model. Invest Ophthalmol Vis Sci 48, 4519-4526, doi:10.1167/iovs.07-0567 (2007).

38 Flynn, T. H. et al. Use of ultrasonic pachymetry for measurement of changes in corneal thickness in mouse corneal transplant rejection. Br J Ophthalmo/ 94, 368-371, doi:10.1136/bjo.2009.160671 (2010).

39 Chauhan, S. K., Jurkunas, U., Funaki, T., Dastjerdi, M. \& Dana, R. Quantification of allospecific and nonspecific corneal endothelial cell damage after corneal transplantation. Eye (Lond) 29, 136-144, doi:10.1038/eye.2014.248 (2015). 
40 Merian, J., Gravier, J., Navarro, F. \& Texier, I. Fluorescent nanoprobes dedicated to in vivo imaging: from preclinical validations to clinical translation. Molecules 17, 5564-5591, doi:10.3390/molecules17055564 (2012).

41 Vahrmeijer, A. L., Hutteman, M., van der Vorst, J. R., van de Velde, C. J. \& Frangioni, J. V. Imageguided cancer surgery using near-infrared fluorescence. Nat Rev Clin Oncol 10, 507-518, doi:10.1038/nrclinonc.2013.123 (2013).

42 Cordeiro, M. F. et al. Real-time imaging of single neuronal cell apoptosis in patients with glaucoma. Brain 140, 1757-1767, doi:10.1093/brain/awx088 (2017).

43 Pels, E., Beele, H. \& Claerhout, I. Eye bank issues: II. Preservation techniques: warm versus cold storage. Int Ophthalmol 28, 155-163, doi:10.1007/s10792-007-9086-1 (2008).

44 Vinegoni, C. et al. Real-time high dynamic range laser scanning microscopy. Nat Commun 7, 11077, doi:10.1038/ncomms11077 (2016).

45 Hertsenberg, A. J. et al. Corneal stromal stem cells reduce corneal scarring by mediating neutrophil infiltration after wounding. PLoS One 12, e0171712, doi:10.1371/journal.pone.0171712 (2017).

46 Percie du Sert, N. et al. The ARRIVE guidelines 2.0: Updated guidelines for reporting animal research. PLoS Bio/ 18, e3000410, doi:10.1371/journal.pbio.3000410 (2020).

47 Peh, G. S. L. et al. Propagation of Human Corneal Endothelial Cells: A Novel Dual Media Approach. Cell Transplantation 24, 287-304, doi:10.3727/096368913×675719 (2015).

48 Bhogal, M., Maurino, V. \& Allan, B. D. Use of a single peripheral triangular mark to ensure correct graft orientation in Descemet membrane endothelial keratoplasty. J Cataract Refract Surg 41, 2022-2024, doi:10.1016/j.jcrs.2015.08.005 (2015).

49 Bhogal, M. S. \& Allan, B. D. Graft profile and thickness as a function of cut transition speed in Descemet-stripping automated endothelial keratoplasty. J Cataract Refract Surg 38, 690-695, doi:10.1016/j.jcrs.2011.09.046 (2012).

\section{Figures}



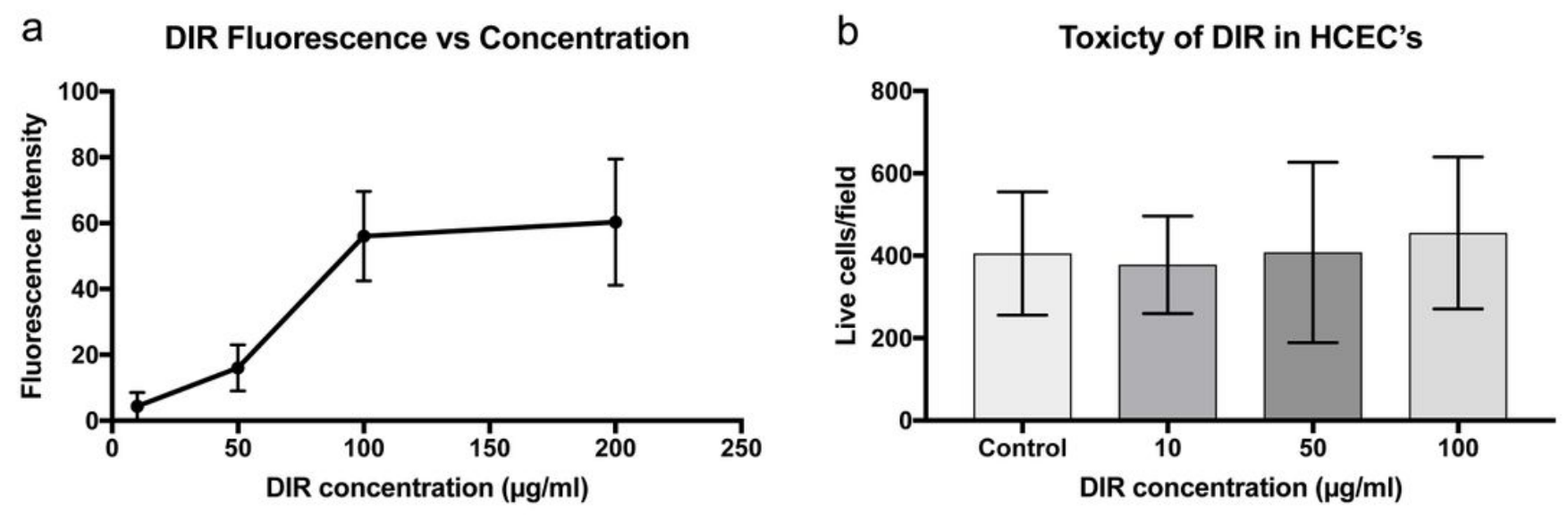

Figure 1

Optimization of DIR concentration in vitro. (A) Graph showing DIR fluorescence in human CECs as a function of concentration. (B) Bar graph comparing the toxicity of DIR at different concentrations. 

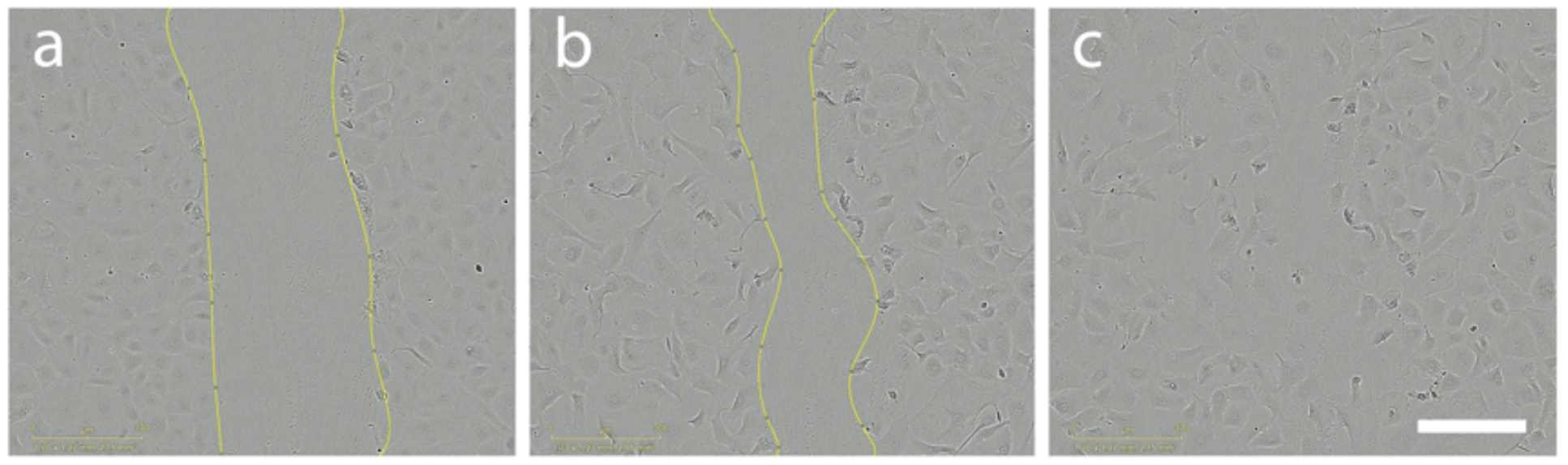

d

\section{Wound Healing kinetics}

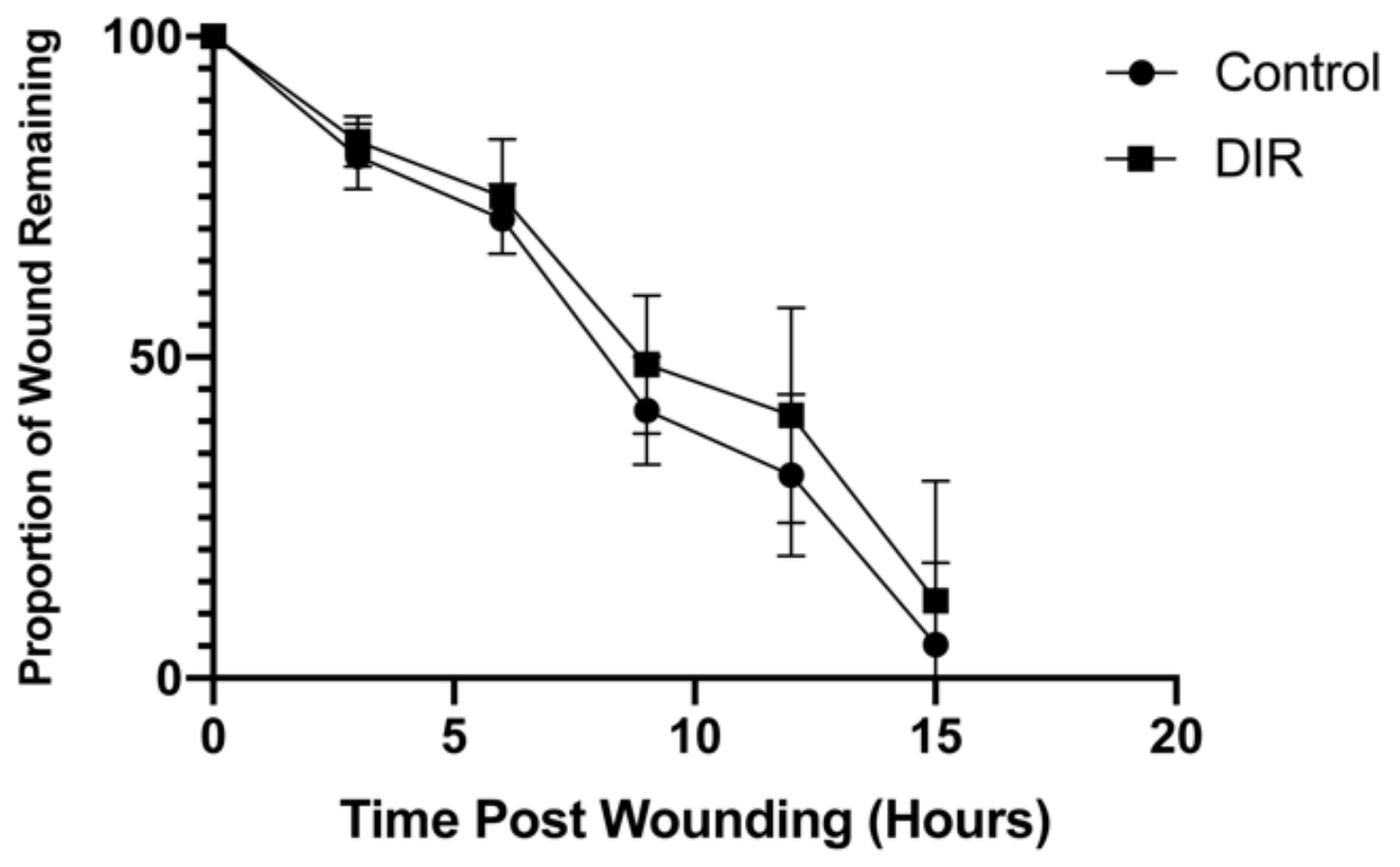

Figure 2

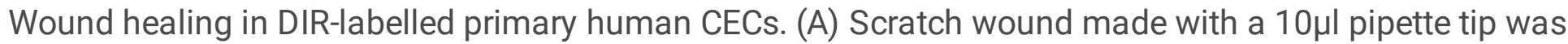
delineated and the wound area measured (yellow border). (B) The wound area was reassessed at 12 hours and the percentage wound area remaining calculated. (C) Time-lapse imaging taken till the timepoint at which complete closure of the wounds was determined. Scale bar, $250 \mu \mathrm{m}$. 

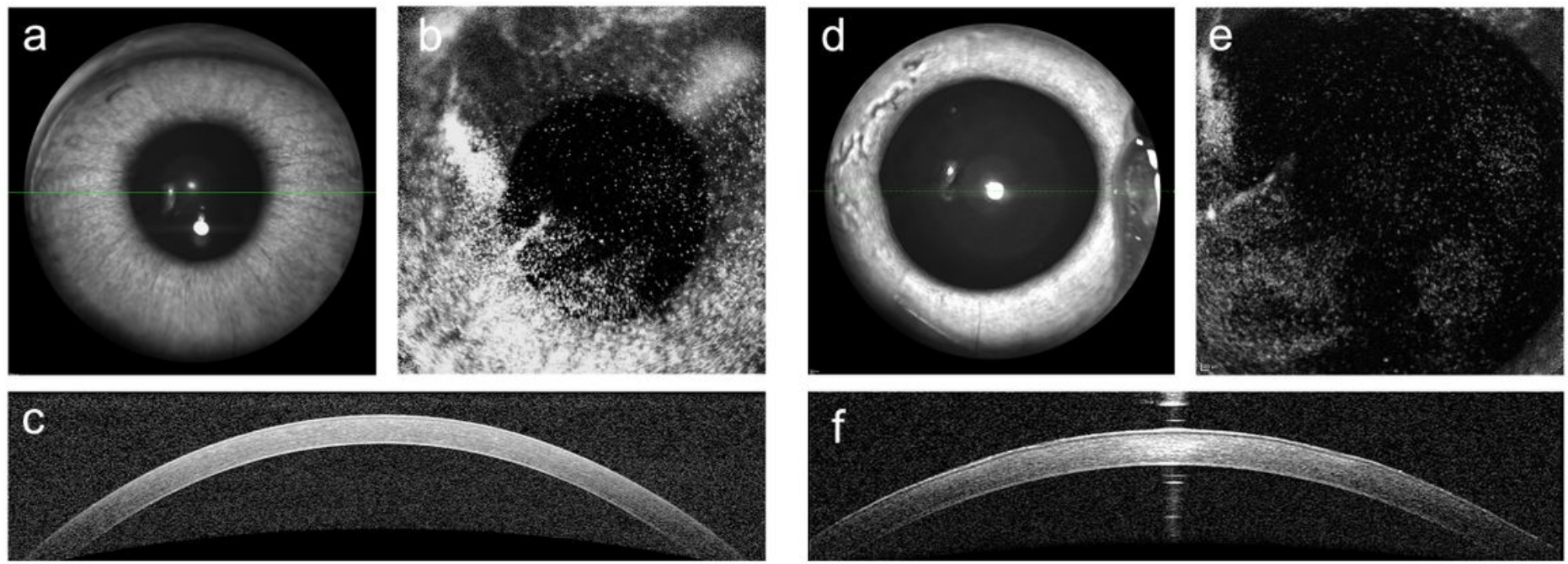

Figure 3

The effect of DIR on rabbit corneal endothelium following intracameral injection in vivo. (A) Day 1 post DIR intracameral injection shows no reduction in corneal clarity. (B) Endothelial cells show variable fluorescence. (C) No corneal thickening or inflammation is seen. (D) At day 28 the cornea remains clear. (E) Residual fluorescence is seen, although maximal fluorescence intensity is reduced. (F) Corneal thickness remains normal throughout the study period. 


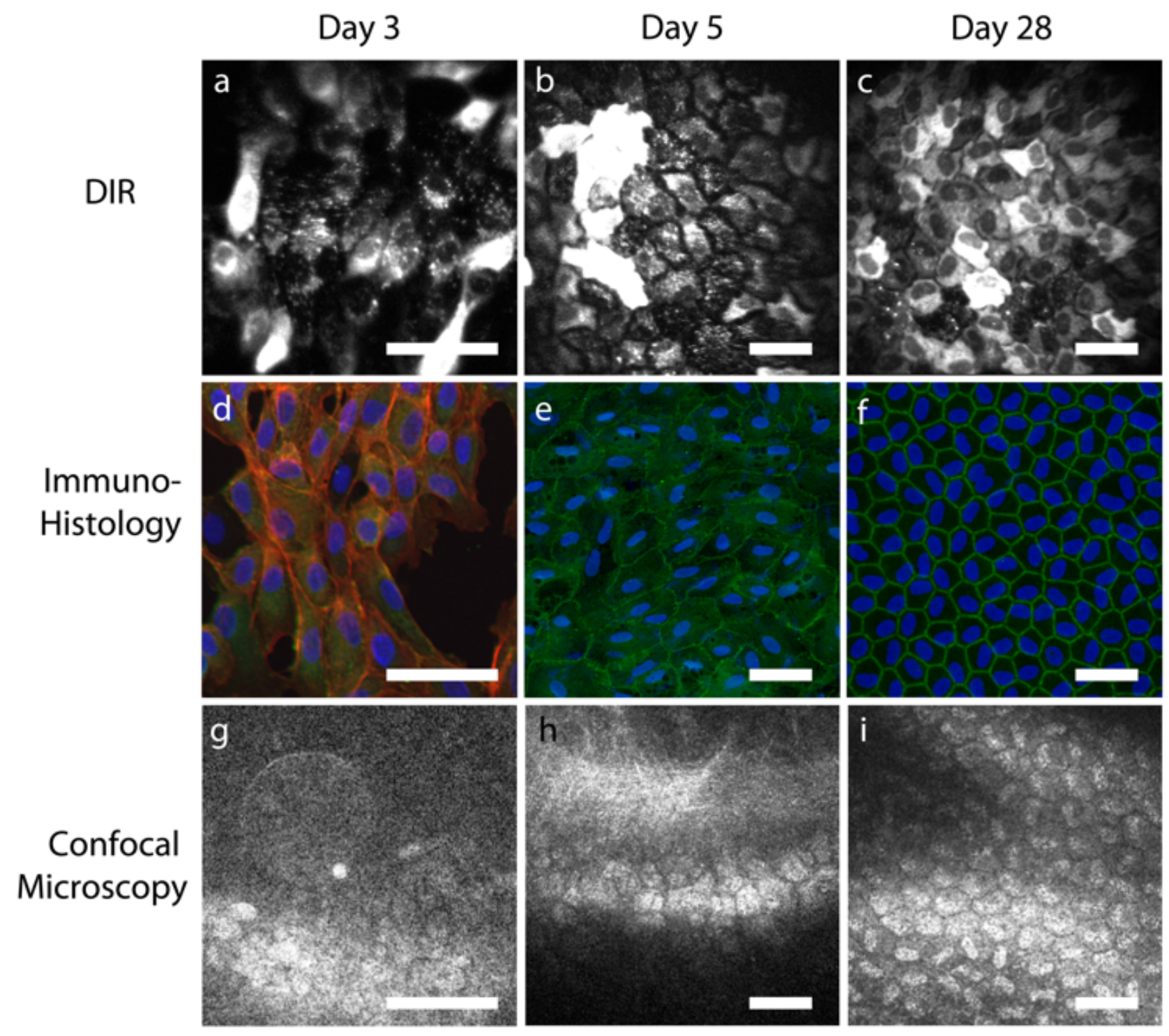

Figure 4

In vivo tracking of corneal endothelial cells. The superior row shows cells at day 3,5 and 28 post wounding as seen by in vivo DIR staining. The middle row shows the same cells imaged with immunohistology at the same time points. The bottom row shows images acquired with conventional in vivo confocal microscopy. (A) In vivo DIR imaging at day 3 showing migrating endothelial cells at the leading edge of the wound. Cells show an elongated morphology with incomplete monolayer formation. (B) At day 5 a complete monolayer is seen with high degrees of pleomorphism and broad intercellular junctions. (C) By day 28 cells have regained a more regular morphology and tight junctions have become narrower. Fixed cells stained with phalloidin, ZO-1 and Hoechst show similar morphological features to in vivo DIR imaging. (D) At day 3 cells are elongated with no junctional ZO-1 staining. (E) At day 5 a complete monolayer is seen with incomplete junctional ZO-1 staining and pleomorphic cells. (F) At day 
28 , regular cells with mature borders are seen. $(G)$ Conventional in vivo confocal imaging failed to acquire useful images at day 3 , and $(\mathrm{H})$ day 5 . (I) At day 28 a stable monolayer of cells was visible on conventional confocal microscopy, but nuclear morphology was more prominent than cell border morphology. Scale bar, $100 \mu \mathrm{m}$.
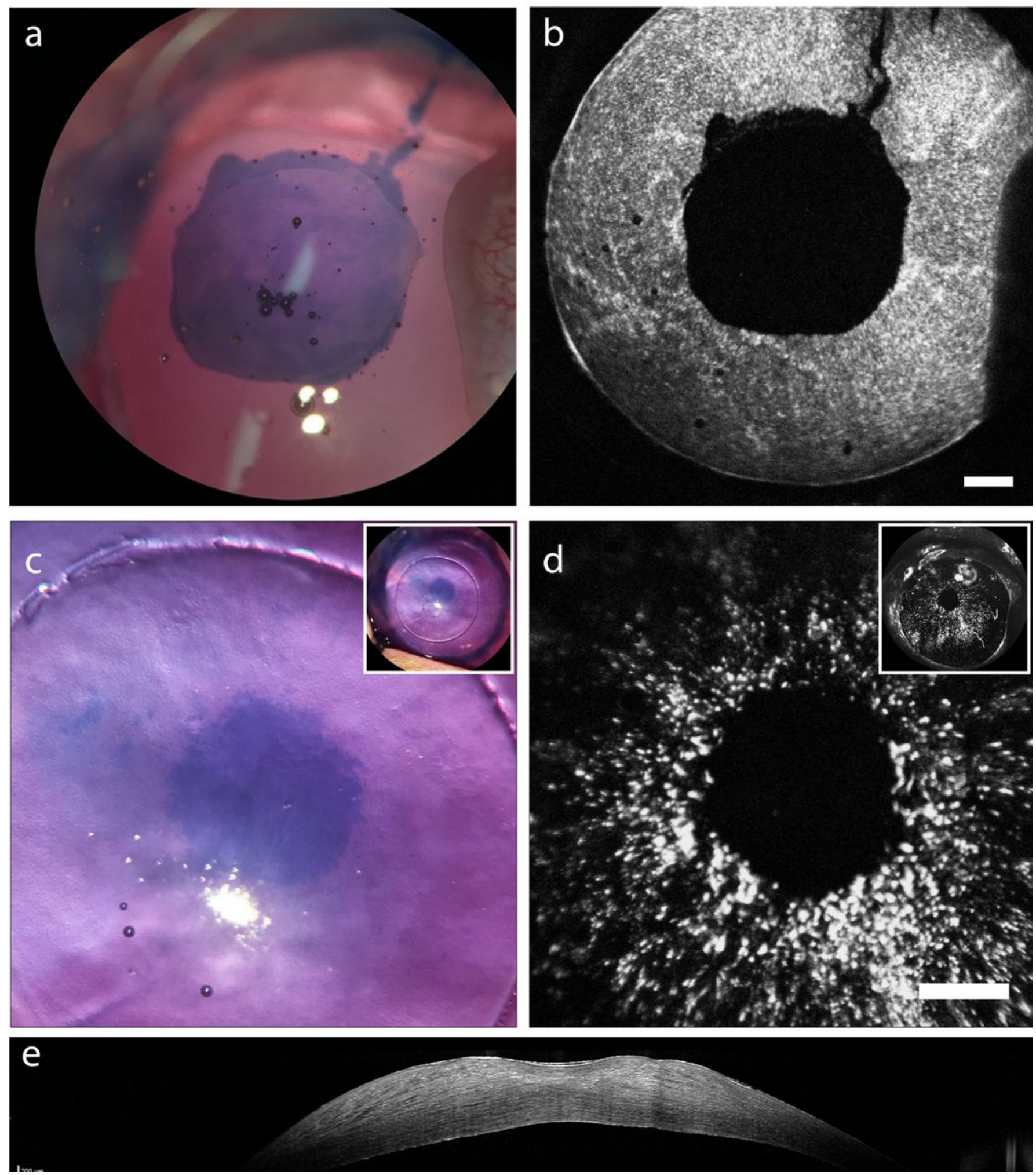

Figure 5 
Comparison between in vivo DIR imaging and trypan blue staining. (A) Intraoperative trypan blue staining shows bare DM where endothelial cells, previously stained with intracameral DIR injection, have been removed by scraping. (B) The same eye was imaged immediately post-operatively. The DIR imaging of the area of denuded endothelial cells shows good concordance with intraoperative trypan blue. DIR fluorescence is blocked by the nictitating membrane. (C) At day 3 post wounding, a small residual area of trypan blue staining is seen. A feathered edge is observed, representing migrating endothelial cells. (D) Good concordance with non-invasive DIR imaging is seen. (E) OCT image of the cornea imaged at day 3 post wounding confirms DIR images are successfully acquired through thickened and irregular corneas. Scale bar, $1 \mathrm{~mm}$.

\section{Bland Altman Plots Comparing DIR and Trypan Blue Imaging}

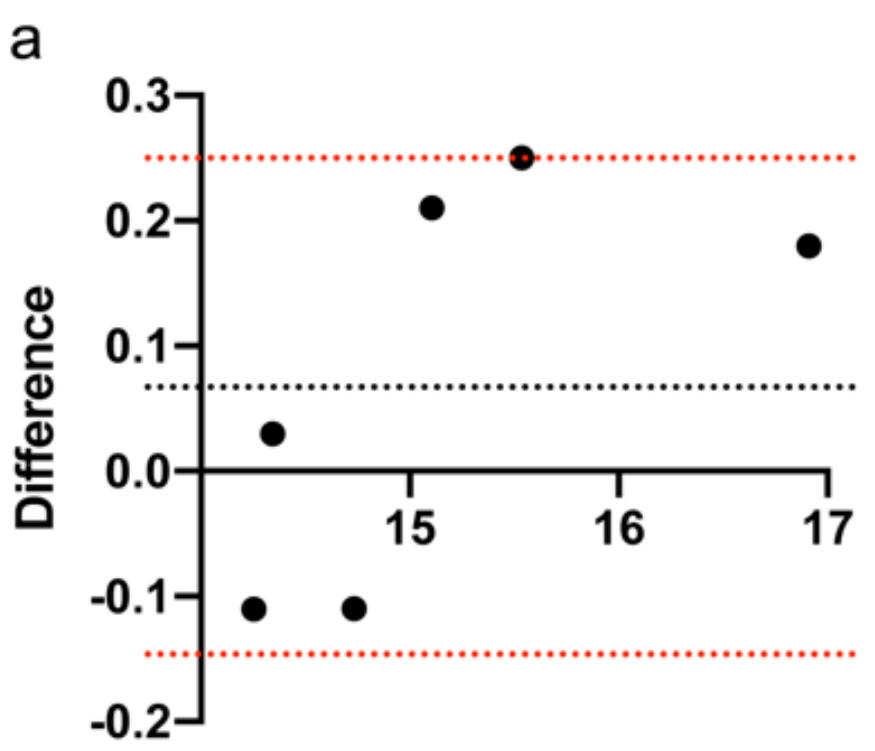

Average Area $\mathrm{mm}^{2}$ b

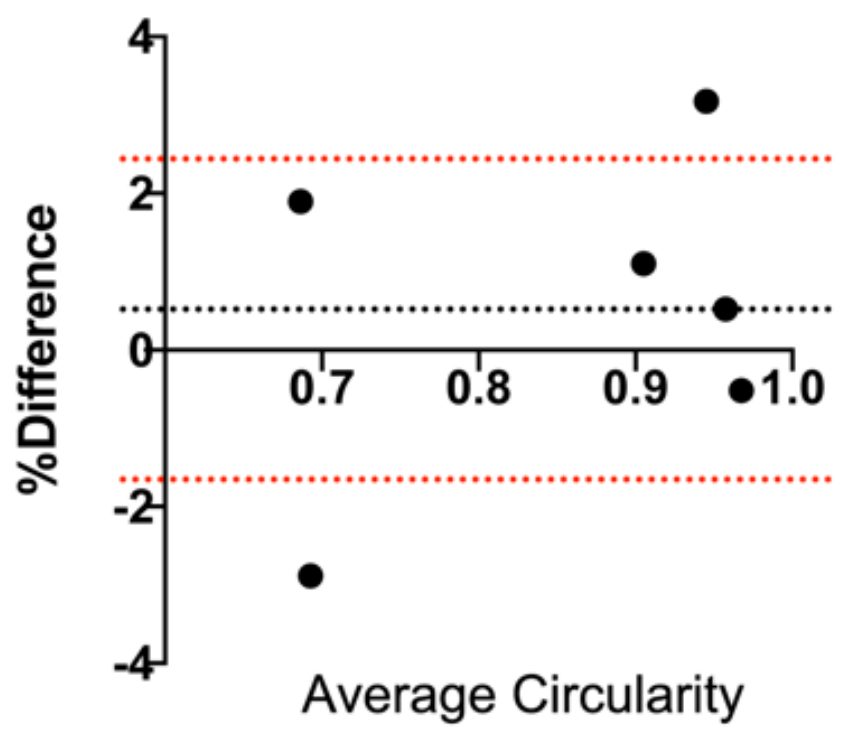

Figure 6

Bland-Altman Plots comparing in vivo wounds assessed DIR staining and intra-cameral trypan blue injection. Dashed black line represents the bias, red dashed lines represent one standard deviation. (A) Average area was within $0.3 \mathrm{~mm}$ for all samples between trypan blue and DIR (B) Average circularity was within $4 \%$ for all samples between trypan blue and DIR. 


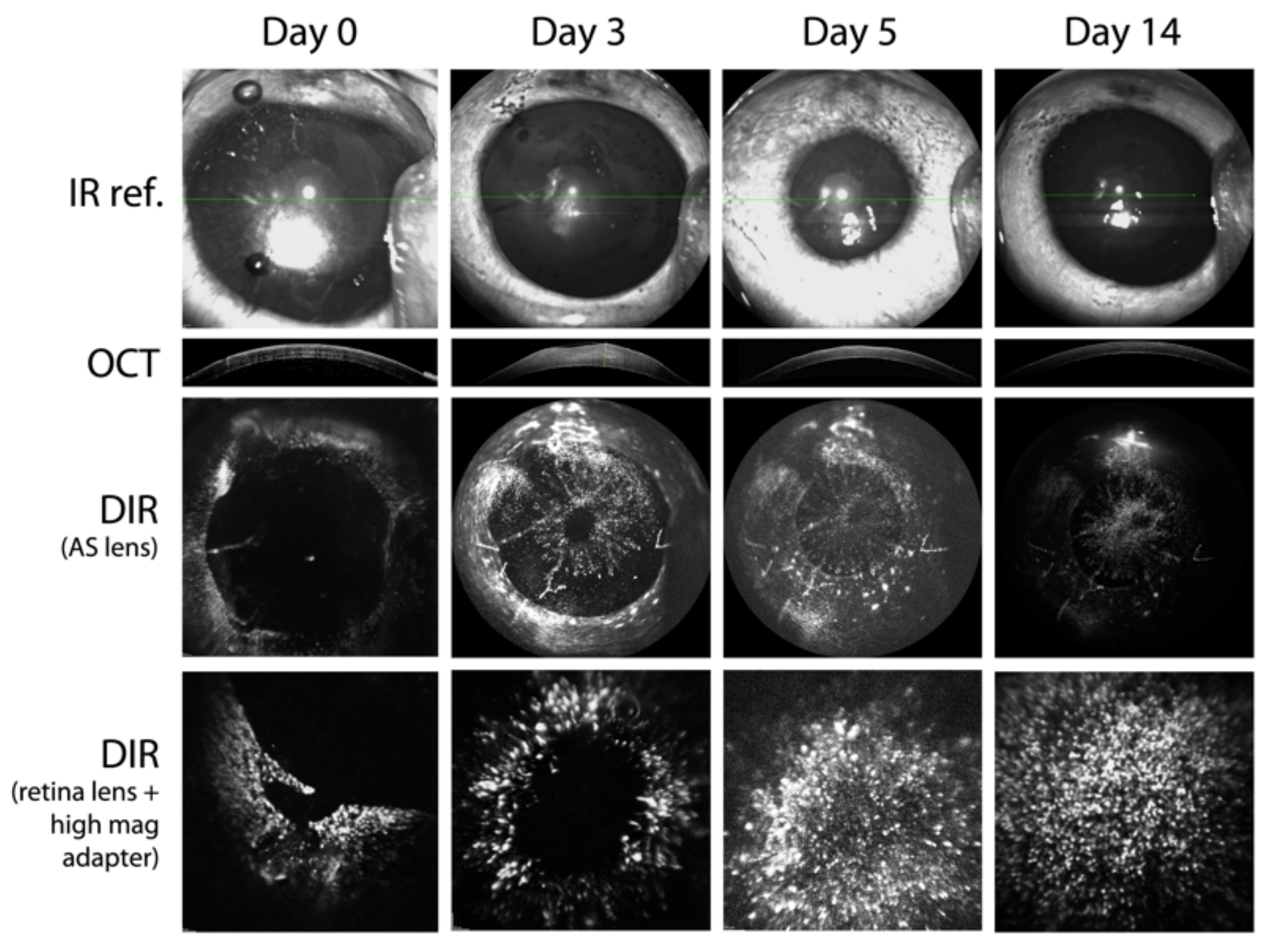

Figure 7

In vivo tracking of endothelial cells at pan-corneal and single-cell level. Following wounding of rabbit corneal endothelium in vivo, tracking of endothelial cells can be conducted at the pan-corneal and singlecell level. Migrating cells have not formed a complete monolayer by day 3. By day 5 complete wound closure has occurred, although residual corneal oedema persist. By day 14 corneal thickness had returned to normal and a stable, healed wound was observed with macroscopic imaging. 


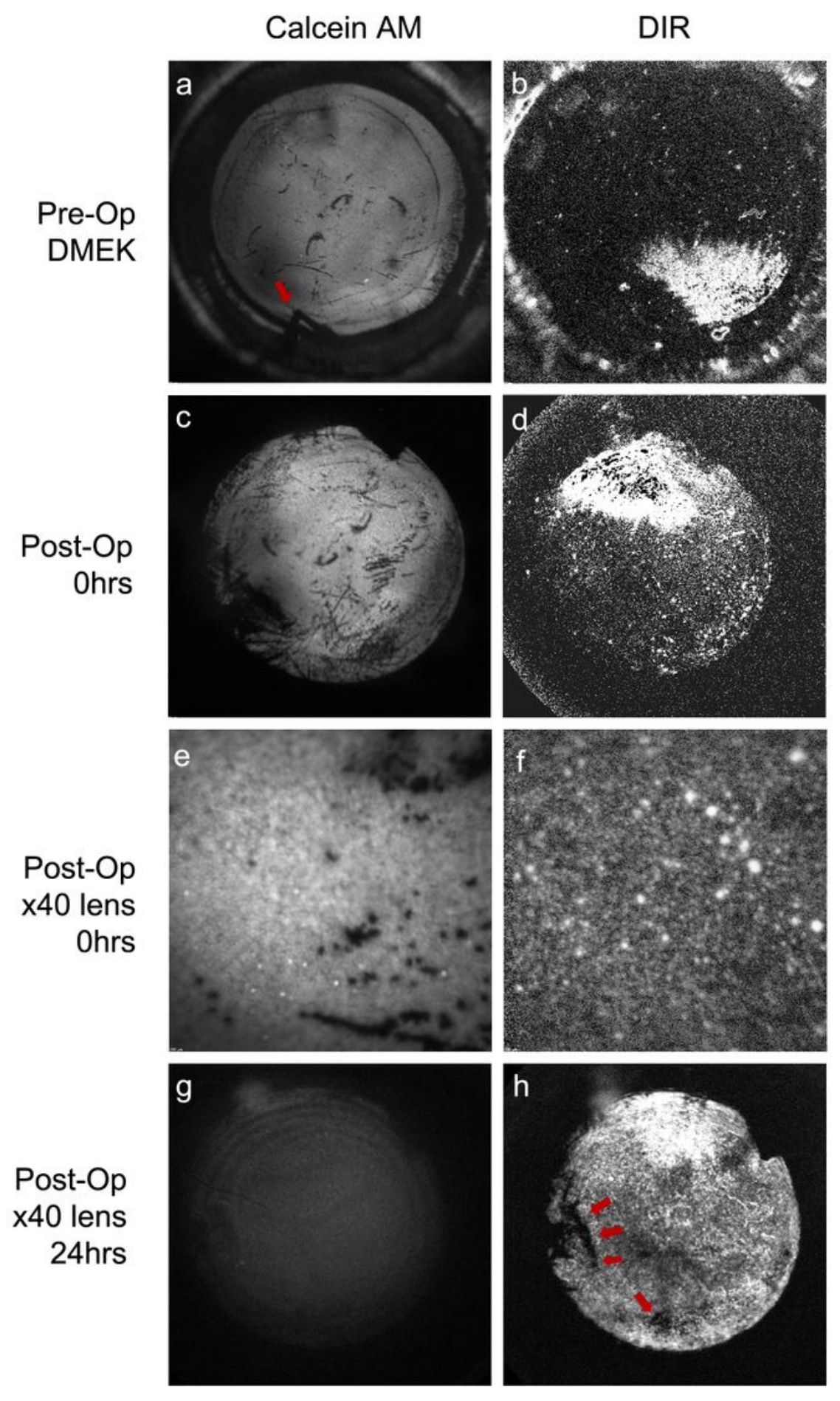

\section{Figure 8}

Labelling of ex vivo human corneas. (A) Prepared DMEK graft resting on stroma. Note triangular orientation mark (red arrow). Uniform green fluorescence is seen. (B) The same transplant is imaged with using the ICG filters to detect DIR staining. There is marked variation in fluorescence across the graft meaning portions become wither over or under exposed on global imaging. (C) Global Calcien AM imaging of the graft, in vivo, immediately following transplantation. (D) Global DIR imaging of the graft, 
in vivo, immediately following transplantation. (E) High magnification imaging acquired by coupling a x40 air objective microscope lens to the retinal imaging lens of the Spectralis. Individual Calcein AM negative cells are seen. (F) DIR imaging at this stage is insufficient to differentiate living and dead cells. (G) At 24 hours post transplantation Calcein AM fluorescence has diminished meaning it is not possible to distinguish between portions of the graft containing viable and non-viable endothelial cells. $(\mathrm{H}) \mathrm{By}$ contrast, at 24 hrs post-implantation, DIR fluorescence has become more uniform and macroscopic areas corresponding to cell damage or missing cells are discernible (red arrows). Scale bar, $1 \mathrm{~mm}$.

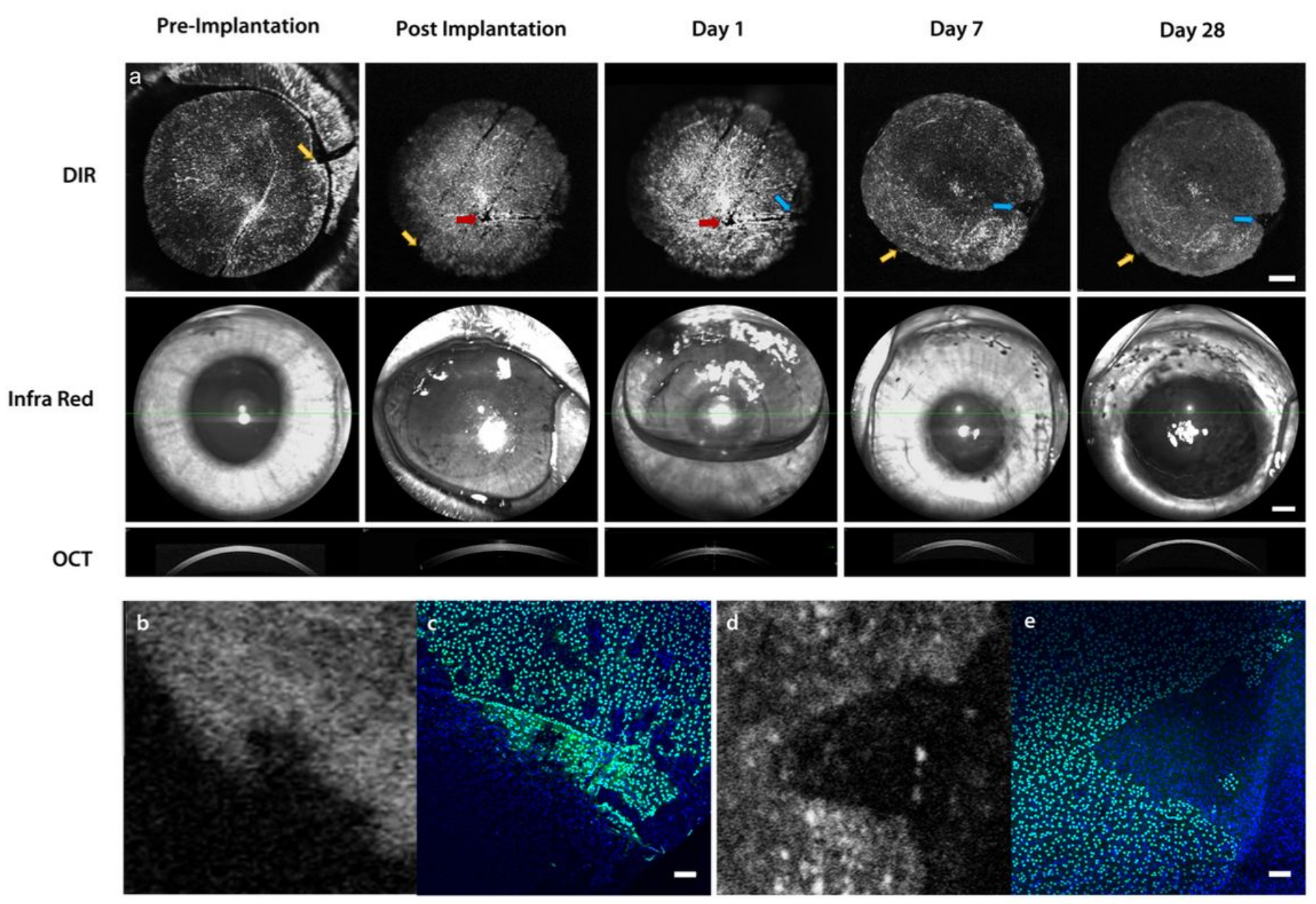

Figure 9

Tracking of transplanted human corneal endothelial cells in vivo. (A) Linear, parallel wounds associated with graft insertion can be seen following DMEK (red arrow). Over time healing of these wounds can be observed. Human cell migration off the transplant and onto the rabbit DM can be seen (yellow arrow) in certain areas. Stable areas, not containing human cells, are visible in the periphery of the graft (blue arrow). Scale bar, $1 \mathrm{~mm}$. (B, C) Immunohistological assessment with anti-HuNu antibody confirms areas of DiR staining adjacent to the graft correspond to Human endothelial cells. Scale bar, $250 \mu m,(D, E)$ Areas on the graft periphery not staining with DIR contain migrating rabbit endothelial cells that form a 
stable monolayer with adjacent human cells, with no significant transfer of fluorescence. Scale bar, $250 \mu \mathrm{m}$.

\section{Day 21}
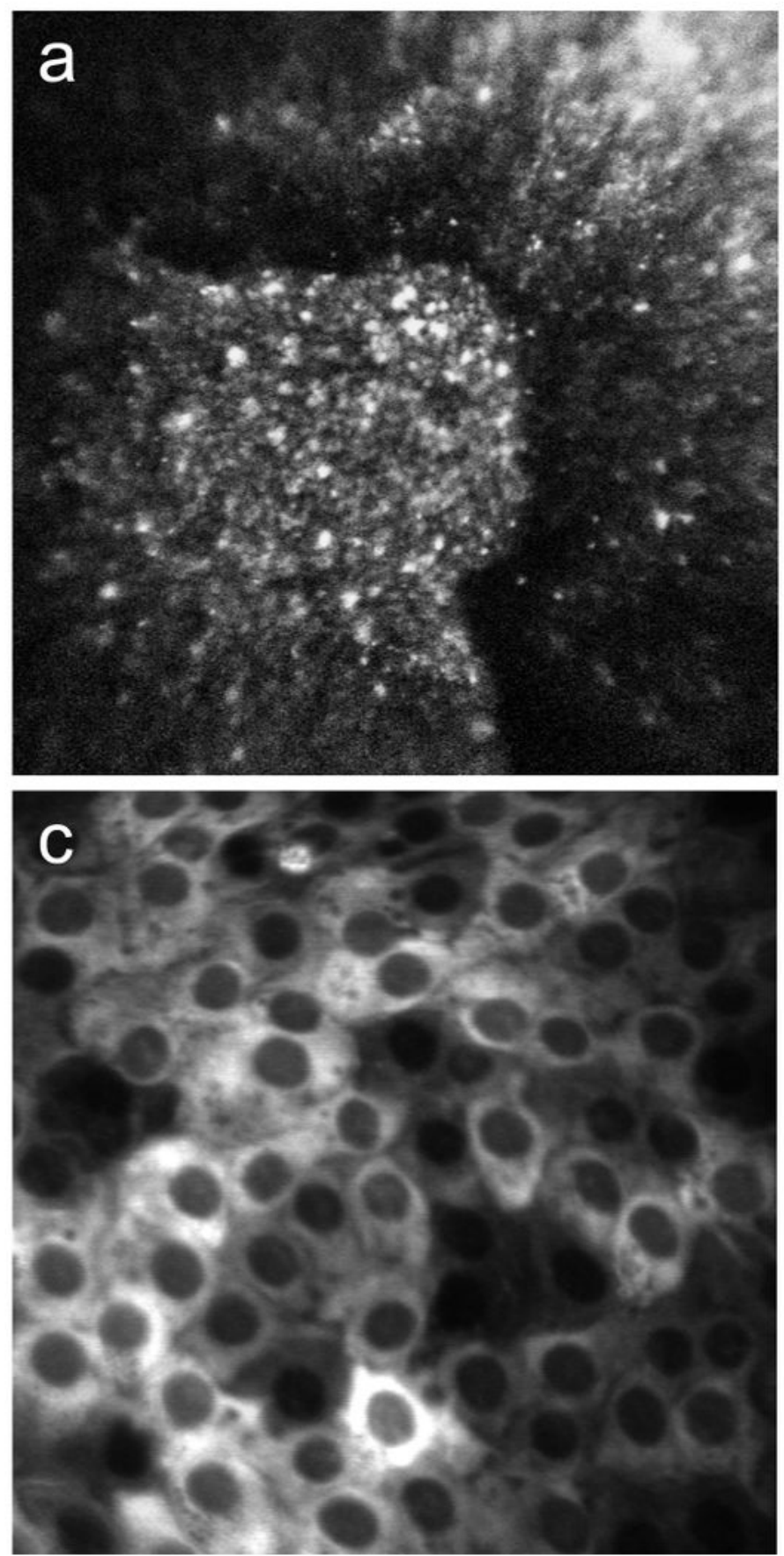

Day 28
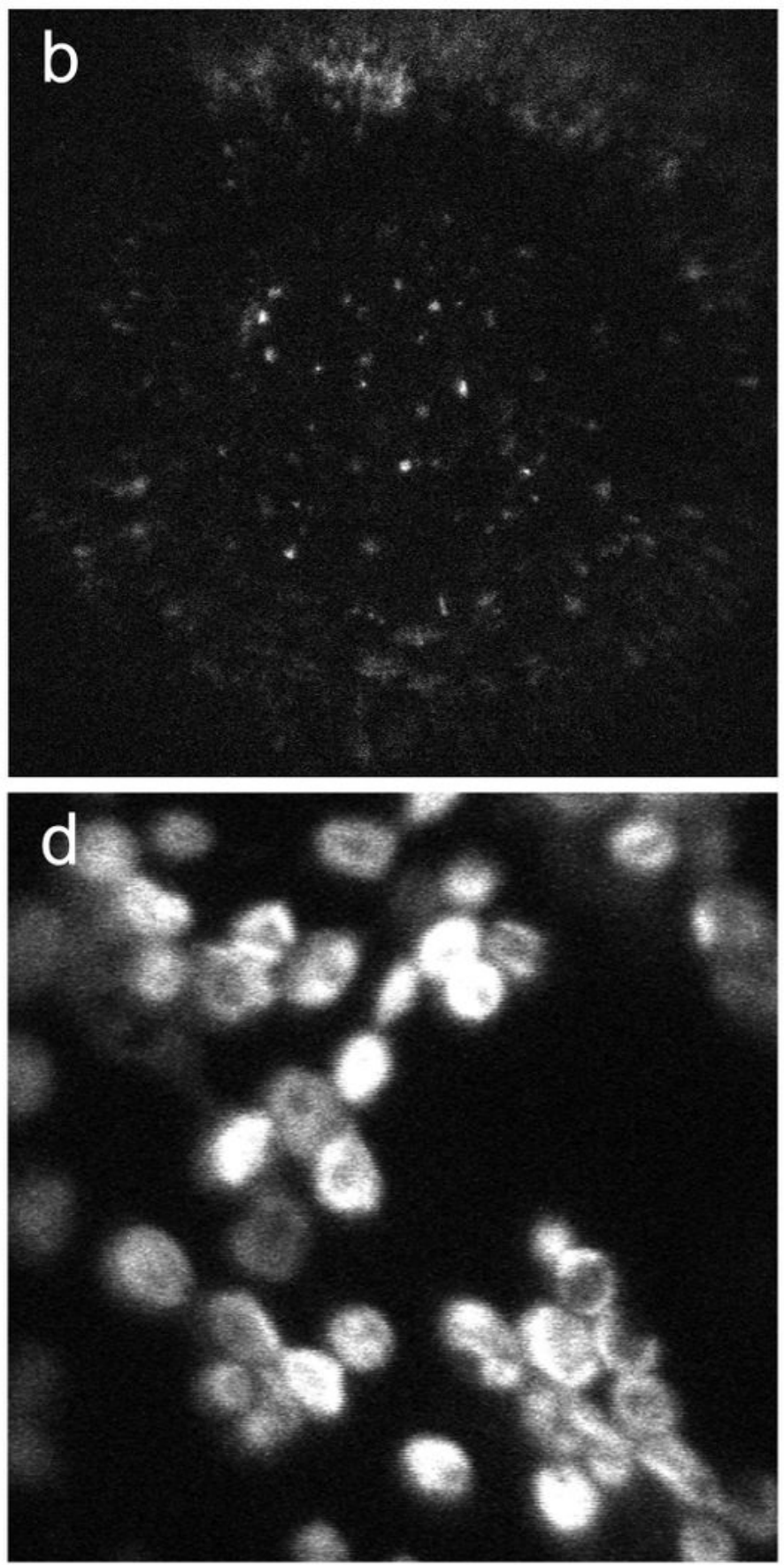

\section{Figure 10}

Spectralis images depicting an event of acute graft rejection. (A) Spectralis HRA image of a central area of a human DMEK graft, 21 days after transplantation into a rabbit eye, shows a confluent monolayer of DIR positive cells. On day 28 corneal oedema was noted, where (B) a significant reduction in DIR 
fluorescence was observed. (C) At higher magnifications, a monolayer of human endothelial cells was seen at day 21. (D) On day 28 , the stable monolayer has been replaced with sporadic cells that appeared rounded and are in the process of detaching from the Descement's membrane.

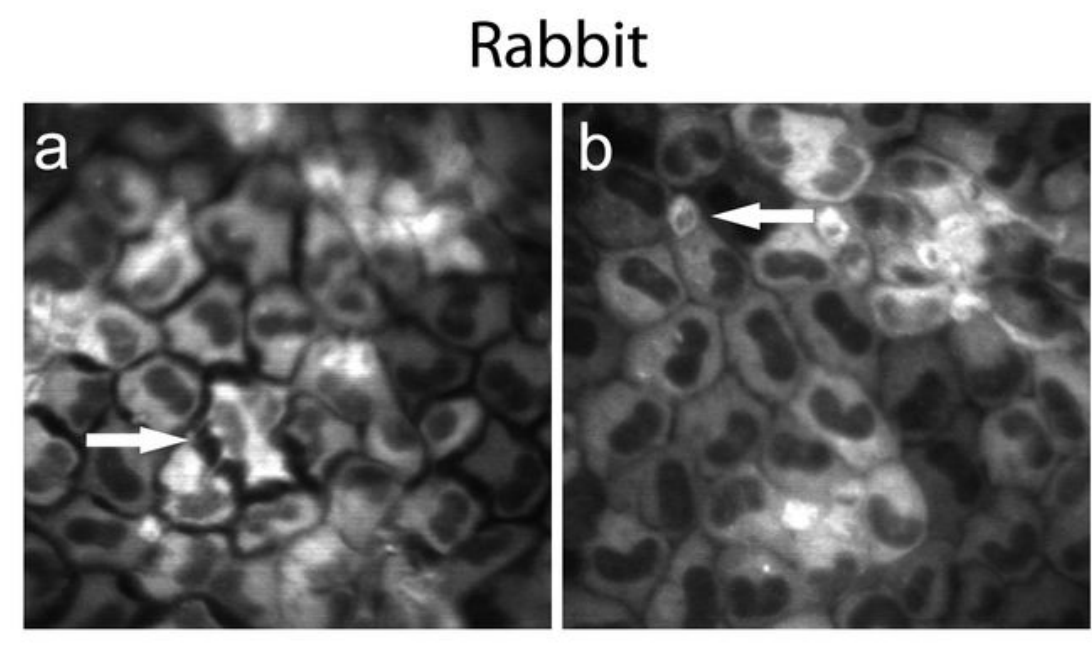

\section{Human}
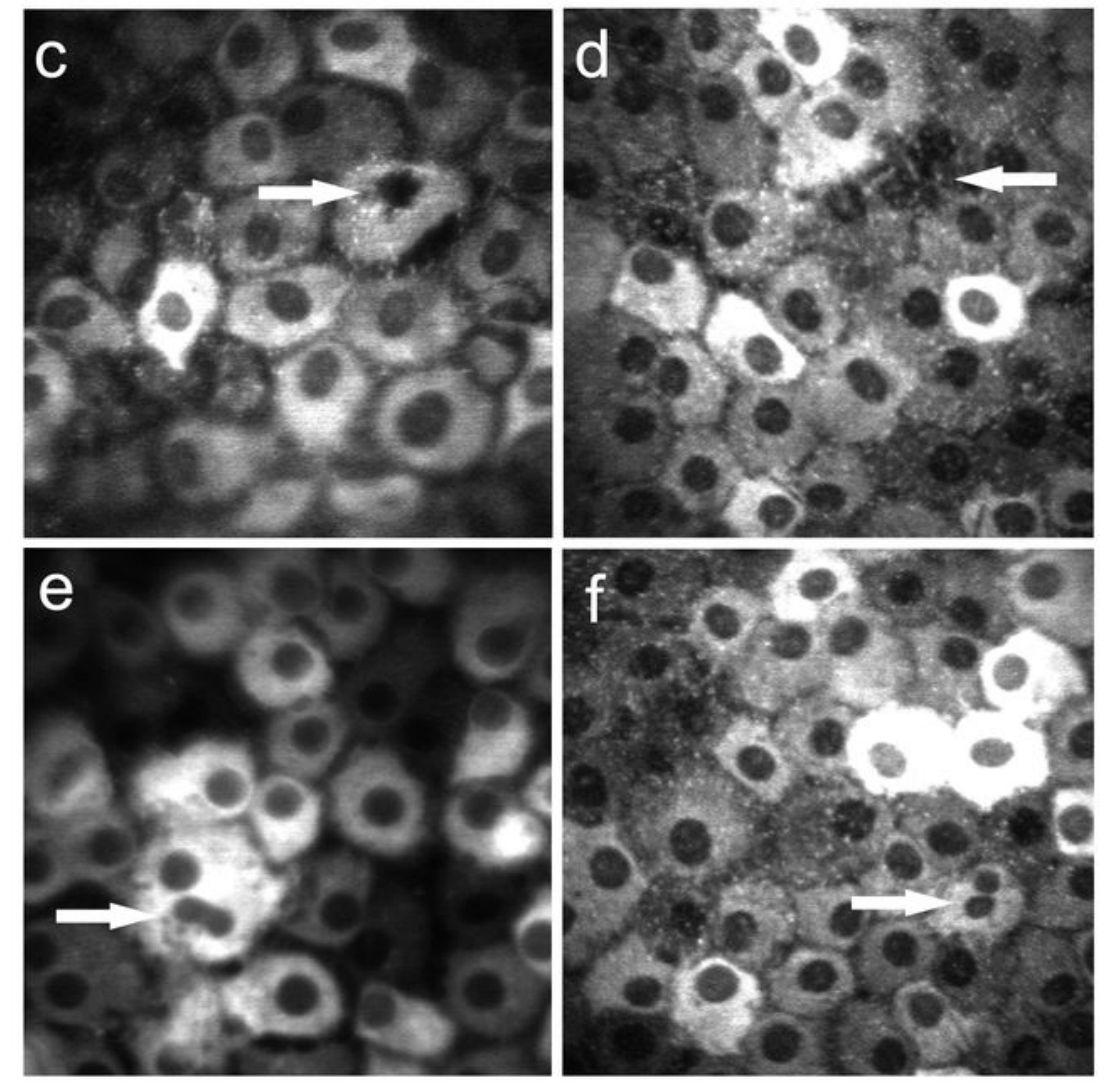

\section{Figure 11}

High-resolution images achieved with Spectralis and Rostock module. High-resolution images of rabbit endothelial cells (stained with DIR by intracameral injection) and human endothelial cells (from EK 
transplants stained with DIR prior to implantation) are achievable with the Spectralis HRA and Rostock module. (A) A rabbit endothelial cell can be seen detaching from the monolayer. (B) Small inflammatory cells staining positive for DIR are visible. (C) A human endothelial cell undergoing apoptosis exhibits a pyknotic nucleus and separation from adjacent cells. (D) Podocyte extension to fill a gap in the monolayer following is seen. (E) Bi-lobulated nuclei and (F) smaller adjacent nuclei suggestive of paired daughter cells are suggestive of in vivo cell division. 